\title{
A STUDY OF CORRELATION OF VISUAL FIELD CHANGES BY HUMPHREY'S AUTOMATED FIELD ANALYSER WITH RETINAL NERVE FIBER LAYER (RNFL) DEFECTS ON OPTICAL COHERENCE TOMOGRAPHY (OCT) IN PRIMARY OPEN ANGLE GLAUCOMA (POAG)
}

\author{
Rajasekhar Pasumarthy¹, Menda Parni Kumar², Divya Deepthi Syamala ${ }^{3}$
}

${ }_{1}^{1}$ Assistant Professor, Department of Ophthalmology, Guntur Medical College, Guntur, Andhra Pradesh. 2 Professor and HOD, Department of Ophthalmology, Guntur Medical College, Guntur, Andhra Pradesh. ${ }^{3}$ Senior Resident, Department of Ophthalmology, Guntur Medical College, Guntur, Andhra Pradesh.

\section{ABSTRACT}

\section{BACKGROUND}

Glaucoma is a significant global health problem. Glaucoma is second only to cataract as a cause of blindness worldwide. As of the year 2000, 67 million people worldwide were affected with "primary" glaucoma. Of these patients, 6.7 million were bilaterally blind. ${ }^{1}$ According to various studies, the prevalence of glaucoma in India ranges from $2.6 \%$ to $4.15 \%{ }^{2}$ It is estimated that there will be 60.5 million people with OAG and ACG in 2010, increasing to 79.6 million by 2020, and of these $74 \%$ will have OAG.

The aim of the study was to correlate the RNFL changes with visual field defects in Primary Open Angle Glaucoma (POAG).

\section{MATERIALS AND METHODS}

It was undertaken in a sample of 52 patients who attended the Ophthalmology OPD, GGH, Guntur. All the patients underwent a comprehensive ocular examination, i.e. Visual acuity recording, Slit lamp examination, Gonioscopy, IOP with Goldmann Applanation Tonometry, Fundus examination with +78D, +90D lens, recording of visual fields on Humphrey's Automated Field Analyser, RNFL analysis with Spectral Domain OCT (optovue) and diagnosed as having POAG in one or both eyes. And the correlation between the RNFL changes and the visual field defects was studied. The results were analysed by using MS Excel 2010 and SPSS (Version 16; SPSS, Chicago) and appropriate tests of statistical significance like Pearson linear regression analysis and unpaired t-test were applied wherever appropriate.

\section{RESULTS}

Linear regression analysis was done between MD and average RNFL thickness, PSD and average RNFL thickness, superior hemifield retinal sensitivity and inferior RNFL thickness and also between inferior hemifield sensitivity and superior RNFL thickness and Pearson correlations ( $r$ value) were $-0.6 .90,0-.519,+0.637$ and +0.665 respectively. From the $r$ values it is seen that as the mean deviation and pattern standard deviation increase, there is a progressive decline in RNFL thickness and the retinal sensitivity decreases, there is a progressive reduction in average RNFL thickness.

\section{CONCLUSION}

Hence, it is concluded that there is positive correlation between visual field defects and RNFL thickness and OCT can be used to assess the severity of glaucoma and also to assess the progression.

\section{KEYWORDS}

Primary Open Angle Glaucoma, Retinal Nerve Fiber Layer, Visual Field Defects, OCT, Humphrey’s Visual Field Analyser.

HOW TO CITE THIS ARTICLE: Pasumarthy R, Kumar MP, Syamala DD. A study of correlation of visual field changes by Humphrey's automated field analyser with retinal nerve fiber layer (RNFL) defects on optical coherence tomography (OCT) in primary open angle glaucoma (POAG). J. Evolution Med. Dent. Sci. 2017;6(26): 2169-2173, DOI: 10.14260/Jemds/2017/470

\section{BACKGROUND}

Glaucoma is characterised by progressive degeneration of retinal ganglion cells and their axons that lead to nerve fiber layer loss, optic disc cupping and consecutive glaucomatous visual field changes and among these retinal nerve fiber layer (RNFL) loss is considered an early sign of glaucoma. ${ }^{3}$ Glaucoma patients are frequently unaware of their disease.

Financial or Other, Competing Interest: None.

Submission 13-03-2017, Peer Review 25-03-2017,

Acceptance 27-03-2017, Published 30-03-2017.

Corresponding Author:

Dr. Divya Deepthi Syamala,

D/o S. Venkateswara Reddy,

Opposite H.I.G 108, Divya Gardens,

Regi Thota, APHB Colony,

Guntur-522005,

Andhra Pradesh.

E-mail: Sdd332@gmail.com

DOI: $10.14260 /$ jemds $/ 2017 / 470$

\section{(c) $(1) \Theta$}

Indeed, glaucoma is often referred to as the "sneak thief of sight." 3 In most industrial societies, more than $50 \%$ of those affected are unaware of their condition. ${ }^{1}$ The percentage of undiagnosed glaucoma exceeds this value in third world nations. According to the Thessaloniki eye study, 57\% of primary open angle glaucoma patients were previously undiagnosed due to lack of regular visits to an ophthalmologist, a major reason for underdiagnosis. ${ }^{4}$

Because injury due to glaucoma is largely irreversible, early detection and prevention of glaucomatous damage is crucial. Examination of the Optic Nerve Head (ONH) and peripapillary RNFL is essential in detecting and monitoring the disease. Recent advances in imaging technologies using the optical properties of the RNFL allow an objective and quantitative assessment of the RNFL thickness. It has been shown that damage to the Retinal Nerve Fiber Layer (RNFL) precedes visual field loss. Quigley et al reported that up to $40 \%$ to $50 \%$ of the RNFL could be lost before visual field defects are detected by conventional perimetry. Thus, RNFL 
assessment has emerged as an important parameter for preperimetric diagnosis of glaucoma and may aid ophthalmologists in making an accurate and early diagnosis.

OCT computes RNFL thickness measurements and is able to identify diffuse and focal RNFL defects reproducibly that occur in glaucoma that have been shown to correlate quantitatively with Visual Field (VF) abnormalities. Both macular and peripapillary RNFL thicknesses as measured by OCT show statistically significant correlation with glaucoma; nevertheless, the peripapillary RNFL thickness turns out to be the best marker in glaucoma assessment.

Hence, this study is undertaken with an aim to measure the RNFL thickness in established cases of glaucoma and to correlate the findings with global indices of visual fields, which would help in detection, in decision making about management and also helps in prognostication of glaucoma.

\section{Methodology}

The current study is an observational cross-sectional study conducted in a sample of 52 patients attending the Outpatient Department, Government General Hospital, Department of Ophthalmology, Guntur Medical College, Guntur, Andhra Pradesh.

\section{Inclusion Criteria}

- $\quad$ Patients of age above 40 yrs.

- $\quad$ IOP > $21 \mathrm{mmHg}$ and Gonioscopically patients with open angles.

- Appearance of optic disc or RNFL suggestive of glaucomatous damage.

- Asymmetric cupping between the two eyes and Visual fields suggestive of glaucomatous damage.

\section{Exclusion Criteria}

- Patients previously diagnosed to have glaucoma and on medication, Angle closure glaucoma, secondary glaucomas.

- Patients with history of other intraocular diseases and complicated intraocular surgery.

- Other diseases affecting visual fields, e.g. pituitary lesions, demyelinating diseases and other neurological diseases.

\section{MATERIALS AND METHODS}

After obtaining approval of the Institutional Ethics Committee, a written/informed consent was taken from patients in his/her vernacular language. A thorough clinical history was taken regarding chief complaint, duration of disease and any other relevant history.

A complete ophthalmic examination including visual acuity testing, slit lamp examination, IOP measurement using applanation tonometer, gonioscopy and a detailed stereoscopic fundus examination was done.

Automated visual field testing was done in all subjects (Humphrey Field Analyser II, Carl Zeiss Meditec) using the central 30-2, 24-2 threshold program and also by using 10-2 threshold program in patients with central $6^{\circ}$ involvement. Average retinal sensitivity of superior and inferior half of 302, 24-2 print out was calculated and MD, PSD were noted. RNFL analysis was done using spectral domain OCT (OptoVue). The HVF changes were compared with the findings of RNFL in OCT.
The patients were divided into mild, moderate and severe glaucoma based on the mean deviation values in the visual field analysis given by Hodapp et al, 5 (Table no. 1).

The results were analysed by using MS Excel 2010 and SPSS (Version 16; SPSS, Chicago) and appropriate tests of statistical significance like Pearson linear regression analysis and unpaired t-test were applied wherever appropriate.

\section{RESULTS}

In the present prospective analytical study conducted, 84 eyes of 52 patients were included after satisfying the inclusion and exclusion criteria who were attending the Outpatient Department of Government General Hospital, Guntur. All of them underwent HVF 30-2 and 24-2 visual field analysis and based on the mean deviation values, they were divided into 3 groups (mild, moderate, severe) and later all the three groups were further evaluated for RNFL thickness using OCT. The observations analysed using appropriate statistical tests and the results are as obtained.

Age of the patients included in the study ranged between 44 and 78 years. The mean age of patients included in the study is $60.11 \pm 8.41$ years. Male population was more in the present study than female population.

Out of the 84 eyes studied, 11 eyes are classified as having mild glaucoma, 33 eyes as moderate glaucoma and 40 eyes as severe glaucoma.

The average mean deviation of all eyes according to visual field analysis was $16.82 \pm 9.619 \mathrm{~dB}$ of mild glaucoma group was $4.68 \pm 1.286 \mathrm{~dB}$, in moderate glaucoma group was and $9.73 \pm 1.693 \mathrm{~dB}$ and in severe glaucoma group was $26.02 \pm$ $4.812 \mathrm{~dB}$, i.e. with increase in severity of glaucoma mean deviation increases.

The average pattern standard deviation of all eyes according to visual field analysis was $5.72 \pm 3.218 \mathrm{~dB}$, of mild glaucoma group was $2.34 \pm 0.651 \mathrm{~dB}$, in moderate glaucoma group was $4.33 \pm 1.479 \mathrm{~dB}$ and in severe glaucoma group was $7.82 \pm 3.281 \mathrm{~dB}$, which means with increase in severity of glaucoma pattern standard deviation increases.

The average superior hemifield retinal sensitivity of visual field analysis is $24.04 \pm 1.603 \mathrm{~dB}$ in mild glaucoma group, $19.53 \pm 3.565 \mathrm{~dB}$ in moderate glaucoma group and $3.69 \pm 4.424 \mathrm{~dB}$ in severe glaucoma group. The average inferior hemifield retinal sensitivity of visual field analysis is $23.93 \pm 2.027 \mathrm{~dB}$ in mild glaucoma group, $19.41 \pm 3.626 \mathrm{~dB}$ in moderate glaucoma group and is $5.46 \pm 5.812 \mathrm{~dB}$ in severe glaucoma group, i.e. as the severity of glaucoma increases there is gradual reduction in the retinal sensitivity.

The average, superior, inferior, temporal, nasal quadrant RNFL thickness according to RNFL analysis by OCT of total 84 eyes were $77.87 \pm 15.53 \mu \mathrm{m}, \quad 90.38 \pm 16.418 \mu \mathrm{m}$, $102.13 \pm 25.214 \mu \mathrm{m}, 61.41 \pm 15.176 \mu \mathrm{m}, 59.63 \pm 15.405 \mu \mathrm{m}$ respectively. The average RNFL thickness according to RNFL analysis by OCT is $94.09 \pm 3.208 \mu \mathrm{m}$ in mild glaucoma group, $86.21 \pm 9.685 \mu \mathrm{m}$ in moderate glaucoma group and $66.53 \pm$ $12.985 \mu \mathrm{m}$ in severe glaucoma group, i.e. with increase in severity of glaucoma. RNFL thickness progressively decreases and this difference is statistically significant in all quadrants between moderate and severe glaucoma groups.

Linear regression analysis was done between MD and average RNFL thickness, PSD and average RNFL thickness, superior hemifield retinal sensitivity and inferior RNFL thickness and also between inferior hemifield sensitivity and 
superior RNFL thickness and Pearson correlations ( $\mathrm{r}$ value) were $-0.6 .90,0-.519,+0.637$ and +0.665 respectively. From the $R$ values it is seen that as the mean deviation and pattern standard deviation increase, there is a progressive decline in RNFL thickness and the retinal sensitivity decreases, there is a progressive reduction in average RNFL thickness.

Hence, it is concluded that there is positive correlation between visual field defects and RNFL thickness and OCT can be used to assess the severity of glaucoma and also to assess the progression.

\begin{tabular}{|c|c|}
\hline Severity of Glaucoma & Mean Deviation \\
\hline Mild Glaucoma & $<6 \mathrm{~dB}$ \\
\hline Moderate Glaucoma & $6-12 \mathrm{~dB}$ \\
\hline Severe Glaucoma & $>12 \mathrm{~dB}$ \\
\hline
\end{tabular}

Table 1. Classification of Severity of Glaucoma based on Mean Deviation

\begin{tabular}{|c|c|c|c|}
\hline Parameters & $\begin{array}{c}\text { Sujatha Subbaiah } \\
\text { et al }\end{array}$ & $\begin{array}{c}\text { C. Ajtony } \\
\text { et al }\end{array}$ & $\begin{array}{c}\text { Present } \\
\text { Study }\end{array}$ \\
\hline $\begin{array}{c}\text { RNFL with } \\
\text { MD } \\
\text { (r value) }\end{array}$ & +0.051 & -0.718 & -0.690 \\
\hline
\end{tabular}

Table 2. Correlation between MD and Average RNFL Thickness and Comparison with Other Studies

\begin{tabular}{|c|c|c|c|}
\hline Parameters & $\begin{array}{c}\text { Sujatha Subbaiah } \\
\text { et al }\end{array}$ & $\begin{array}{c}\text { C. Ajtony } \\
\text { et al }\end{array}$ & $\begin{array}{c}\text { Present } \\
\text { Study }\end{array}$ \\
\hline $\begin{array}{c}\text { RNFL with } \\
\text { PSD } \\
\text { (r value) }\end{array}$ & -0.355 & -0.689 & -0.519 \\
\hline \multicolumn{3}{|c|}{ Table 3. Correlation between PSD and Average RNFL } \\
Thickness and Comparison with Other Studies \\
\hline
\end{tabular}

\begin{tabular}{|c|c|c|}
\hline Parameters & Choudary et al & Present study \\
\hline $\begin{array}{c}\text { Superior visual field } \\
\text { with inferior RNFL } \\
\text { thickness }\end{array}$ & 0.586 & 0.637 \\
\hline $\begin{array}{c}\text { Inferior visual field } \\
\text { with superior RNFL } \\
\text { thickness }\end{array}$ & 0.451 & 0.665 \\
\hline \multicolumn{2}{|c|}{$\begin{array}{c}\text { Table 4. Correlation of Average RNFL Thickness with } \\
\text { Corresponding Average Retinal Sensitivity (R Value) }\end{array}$} \\
\hline
\end{tabular}

\section{DISCUSSION}

In this current study, 84 eyes of 52 patients were analysed and an attempt has been made to analyse RNFL changes on OCT and visual field defects on Humphrey visual field analysis. Based on mean deviation values patients were divided into 3 groups mild, moderate and severe glaucoma and correlation was attempted between the structural changes on OCT and functional changes on automated visual field analysis printout.

Age of the patients included in the study ranged between 44 and 78 years. The mean age of patients included in the study is $60.11 \pm 8.41$ years. These results coincide with age of onset of primary glaucoma, similar results were found in Leske MC et al. ${ }^{6}$

Males were more in the present study than females. Usually, the prevalence of glaucoma is similar in men and women, but in the present study males were more than females. Such results might have occurred as this being a hospital based study and hospital facilities being availed more by men than women.

Most of the patients in present study were in the age group of $61-70$ yrs.

\section{Visual Field Analysis}

Out of the 84 eyes studied 11 eyes $(13.10 \%)$ were found to have mild glaucoma, 33 eyes $(39.28 \%)$ moderate glaucoma and 40 eyes $(47.62 \%)$ severe glaucoma. The number of patients with mild glaucoma were less compared to other 2 groups, which must have occurred as this is a hospital based study and patients usually present to the hospitals only after there are advanced symptoms.

The average mean deviation value of all 84 eyes of the present study $(16.82 \pm 9.619)$ was more than that of the MD value of C. Ajtony et al $^{7}(4.33 \pm 5.00)$ and Sujatha Subbaiah et $\mathrm{al}^{8}(5.54 \pm 5.582)$ studies. This variation must have occurred due to variation in the sample size included in the 3 studies. One more reason which can be pointed is that in the present study the no. of patients in the mild group were less compared to those with moderate and severe glaucoma, which might have been the reason for occurrence of increased MD value in the present study.

The average pattern of standard deviation of all eyes according to visual field analysis was $5.73 \pm 3.218 \mathrm{~dB}$, of mild glaucoma group was $2.34 \pm 0.651 \mathrm{~dB}$, in moderate glaucoma group was $4.33 \pm 1.479 \mathrm{~dB}$ and in severe glaucoma group was $7,82 \pm 3.281$.

The average PSD value of all 84 eyes of the present study was more than that of the PSD value of C. Ajtony et $\mathrm{al}^{9}(4.05 \pm$ $3.31)$ and Sujatha Subbaiah et $\mathrm{al}^{10}(4.74 \pm 2.82)$ studies. This variation must have occurred due to variation in the sample size included in the 3 studies. One more reason which can be pointed is that in the present study the number of patients in the mild group were less compared to those in moderate and severe glaucoma groups, which might have been the reason for occurrence of increased PSD value in the present study.

The average superior retinal hemifield sensitivity of visual field analysis is $24.04 \pm 1.603 \mathrm{~dB}$ in mild glaucoma group, $19.53 \pm 3.565 \mathrm{~dB}$ in moderate glaucoma group and $3.69 \pm 4.424 \mathrm{~dB}$ in severe glaucoma group.

The average inferior retinal hemifield sensitivity of visual field analysis is $23.93 \pm 2.02 \mathrm{~dB}$ in mild glaucoma group, $19.42 \pm 3.626 \mathrm{~dB}$ in moderate glaucoma group and is $5.46 \pm$ $5.812 \mathrm{~dB}$ in severe glaucoma group.

The above results show that with increase in severity of glaucoma, there is gradual reduction in the retinal sensitivity which is as told by 0 . Punjabi et al. ${ }^{11}$

\section{RNFL Analysis by OCT \\ Average RNFL Thickness}

The average RNFL thickness according to RNFL analysis by OCT of all 84 eyes is $77.87 \pm 15.531 \mu \mathrm{m}$. Similar results were found in C. Ajtony et $\mathrm{al}^{9}(77.99 \pm 17.06)$ study, but not with that of Yalvac IS et al ${ }^{12}(62.90 \pm 16.56)$ and Sujatasubbiah et al $^{10}$ studies.

\section{Average RNFL Thickness in Four Quadrants}

The average, superior, inferior, temporal, nasal quadrant RNFL thickness according to RNFL analysis by OCT of total 84 eyes were $77.87 \pm 15.531 \mu \mathrm{m}, 90.38 \pm 16.41 \mu \mathrm{m}, 102.13 \pm 25.21$ $\mu \mathrm{m}, 61.41 \pm 15.17 \mu \mathrm{m}, 59.63 \pm 15.40 \mu \mathrm{m}$ respectively; follows 
the double hump pattern similar to Sujathasubbiah et al study. ${ }^{10}$

Average RNFL thickness in 4 quadrants, i.e. superior, nasal, inferior and temporal quadrants in patients with mild glaucoma, moderate glaucoma and severe glaucoma was calculated and it shows that with increase in the severity of glaucoma the double hump pattern is progressively flattening but still being maintained.

\section{Correlation between MD and Average RNFL Thickness and Comparison with Other Studies}

The Pearson Correlation value between mean deviation values and average RNFL thickness was calculated in all 84 . A negative correlation was seen between the two and it was significant ( $\mathrm{r}$ value $>0.5$ ), which means as the severity of glaucoma increases there is a progressive decline in average RNFL thickness. Similar results were found in C. Ajtony et al ${ }^{9}$ study and Sihota et $\mathrm{al}^{13}$ study, but not that of Sujatha Subbaiah et $\mathrm{al}^{7}$ study which showed a positive correlation between MD and average RNFL thickness (Table no. 2).

\section{Correlation between PSD and Average RNFL Thickness and Comparison with Other Studies}

The Pearson Correlation value between pattern standard deviation values and average RNFL thickness was calculated in all 84 eyes. A negative correlation was seen between the two and it was significant ( $\mathrm{r}$ value $>0.5$ ), which means as the severity of glaucoma increases there is a progressive decline in average RNFL thickness. Similar results were found in C. Ajtony et $\mathrm{l}^{9}$ study and Sujatha Subbaiah et al ${ }^{10}$ study (Table no. 3).

\section{Correlation of Average RNFL Thickness with Corresponding Average Retinal Sensitivity}

Linear regression analysis was to assess the correlation between superior hemifield retinal sensitivity values of visual field analysis and inferior RNFL thickness of all 84 eyes. The correlation coefficient $r$ was 0.677 , i.e. with decrease in average superior hemifield retinal sensitivity there was a decrease in average inferior RNFL thickness. Similar results were found in Choudary et $\mathrm{al}^{2}$ study $(\mathrm{r}=0.586)$, but $\mathrm{r}$ value was less compared to the present study which might be because no eyes with severe glaucoma are included in the present study than the eyes with mild glaucoma (Table no. 4).

Hence, it is concluded that there is positive correlation between visual field defects and RNFL thickness and OCT can be used to assess the severity of glaucoma and also to assess the progression.

But since this is a hospital-based study with a sample size, the results cannot be generalised to normal population and the number of patients with early glaucoma are less compared to advanced glaucoma. As a positive correlation is seen between visual field analysis and RNFL analysis in the current study, it is recommended that further studies should be carried out in early glaucoma and glaucoma suspects as OCT may aid in earlier detection of disease than visual fields.

\section{CONCLUSION}

From the current hospital based prospective analytical study, the following conclusions are obtained-

- $\quad$ Primary open angle glaucomas are more in advanced age groups.
- Visual field analysis revealed decrease in average superior and inferior retinal hemifield sensitivity with progression of glaucoma.

- Average RNFL thickness, superior hemisphere RNFL thickness, inferior hemisphere RNFL thickness, RNFL thickness in 8 sectors decreased with increase in severity of glaucoma on RNFL algorithms and the difference between the 3 groups was significant.

- There is a negative correlation between average mean deviation and average pattern standard deviation of visual field examination and average RNFL thickness of OCT RNFL analysis.

- $\quad$ There is a positive correlation between average superior hemifield retinal sensitivity and inferior RNFL thickness of OCT RNFL analysis.

- There is a positive correlation between average inferior hemifield retinal sensitivity and superior RNFL thickness of OCT RNFL analysis.

- $\quad$ There is a positive correlation between average retinal sensitivity and average RNFL thickness of OCT RNFL analysis.

- Hence, OCT is not only a tool for glaucoma diagnosis, severity and prognosis, but also its findings of RNFL thickness is corresponding and correlating with the loss of field of vision on HFA.

\section{REFERENCES}

[1] Quigley HA. Number of people with glaucoma worldwide. Br J Ophthalmol 1996;80(5):389-93.

[2] Choudhry RM, Chakraborty T. Comparison of visual field defects and retinal nerve fibre layer analysis by OCT in established cases of glaucoma. in AIOC 2009.

[3] Tuulonen A, Lehtola J, Airaksinen PJ. Nerve fiber layer defects with normal visual fields: do normal optic disc and normal visual field indicate absence of glaucomatous abnormality? Ophthalmology 1993;100(5):587-97; discussion 597-598.

[4] Topouzis F, Wilson MR, Harris A, et al. Prevalence of open-angle glaucoma in Greece: the thessaloniki eye study. Am J Ophthalmol 2007;1(4):511-9.

[5] Hodapp E, Parrish RK, Anderson DR. Clinical decisions in glaucoma. St. Louis: CV Mosby 1993:52-61.

[6] Leske MC, Heijl A, Hussein M, et al. Factors for glaucoma progression and the effect of treatment: the early manifest glaucoma trial. Arch Ophthalmol 2003;121(1):48-56.

[7] Mannasakorn A, Nouri-Mahdavi K, Caprioli J. Comparison of retinal nerve fiber layer thickness and optic disk algorithms with optical coherence tomography to detect glaucoma. Am J Ophthalmol 2006;141(1):105-15.

[8] Vandana B, Mahanaz S, Akbar S, et al. Nerve fiber layer thickness in glaucoma patients with asymmetric hemifield visual field loss. J Glaucoma 2006;15(4):27580 .

[9] Ajtony C, Balla Z, Somoskeoy S, et al. Relationship between visual field sensitivity and retinal nerve fiber layer thickness as measured by optical coherence tomography. IOVS 2007;48(1):258-63. 
[10] Subbiah S, Sankarnarayanan S, Thomas PA, et al. Comparative evaluation of optical coherence tomography in glaucomatous, ocular hypertensive and normal eyes. Indian Journal of Ophthalmology 2007;55(4):283-7.

[11] Punjabi O, Lin S, Stamper R. Advances in mapping the glaucomatous visual field from confrontation to multifocal evoked visual evoked potentials. Internet Journal of Ophthalmology and Visual Sciences 2005;4(1).
[12] Yalvac IS, Altunsoy M, Cansever S, et al. The correlation between visual field defects and focal nerve fiber layer thickness measured with optical coherence tomography in the evaluation of glaucoma. J Glaucoma 2009;18(1):53-61.

[13] Sihota R, Sony P, Gupta V, et al. Diagnostic capability of optical coherence tomography in evaluating the degree of glaucomatous retinal nerve fiber damage. Invest Ophthalmol Vis Sci 2006;47(5):2006-10. 